\title{
Multipoint injection indocyanine green lymphography can reduce lymphaticovenular anastomosis surgical time and improve the surgical results for lymphedema
}

\author{
Gejia Ma, Xin Cui, Yue Han, Wenfei Wang, Bin Liu \\ Department of Burn and Plastic Surgery, Xi'an Central Hospital, Medical School of Xi'an Jiaotong University, Xi'an 710000, \\ Shaanxi, China. \\ Correspondence to: Dr. Gejia Ma, Xi'an Central Hospital, Medical School of Xi'an Jiaotong University, No.185 Houzaimen Road, \\ Xi'an 710000, Shaanxi, China. E-mail: gejiama@hotmail.com; Dr. Bin Liu, Xi'an Central Hospital, Medical School of Xi'an Jiaotong \\ University, No.185 Houzaimen Road, Xi'an 710000, Shaanxi, China. E-mail:leorobbins@163.com
}

How to cite this article: Ma G, Cui X, Han Y, Wang W, Liu B. Multipoint injection indocyanine green lymphography can reduce lymphaticovenular anastomosis surgical time and improve the surgical results for lymphedema. Plast Aesthet Res 2021;8:32. https://dx.doi.org/10.20517/2347-9264.2021.20

Received: 6 Mar 2021 First Decision: 25 Apr 2021 Revised: 9 May 2021 Accepted: 9 Jun 2021 Available online: 4 Jul 2021

Academic Editors: Raúl González-García, Matthew L lorio, Isao Koshima Copy Editor: Yue-Yue Zhang Production Editor: YueYue Zhang

\begin{abstract}
Aim: Lymphaticovenular anastomosis (LVA) is the mainstay of surgical treatment of lymphedema now. Indocyanine green (ICG) lymphography is a method for detecting lymphatic pathways and for the clinical evaluation of patients with extremity lymphedema. The essential point of LVA is to find more functional lymphatic vessels. Sometimes, in cases of lymphatic dysfunction, ICG injections into the distal extremities are insufficient. The purpose of this study was to elucidate the effect of multi-injection of ICG lymphography on LVA.

Methods: In this study, we injected ICG into the second web of the hands or the first web of the feet. In the multiinjection group, we injected additional ICG in other sites. We observed the presence or absence of a linear pattern at each injection site with a near-infrared camera. Then, we performed LVA and evaluated the circumference change and the operation time.
\end{abstract}


Results: In the multi-injection group, we injected ICG into the upper limb at an average of 2.2 sites (range: 1-3 sites) and the lower limb at an average of 3.2 sites (range: 1-5 sites). The circumference change of upper limbs in the control group was $-3.95 \% \pm 1.34 \%$ and in the multi-injection group was $-6.96 \% \pm 0.88 \%(P<0.05)$. The change in circumference of lower limbs was $-5.01 \% \pm 2.2 \%$ in the multi-injection group and $-2.33 \% \pm 1.77 \%$ in the control group $(P=0.003)$. The mean surgical duration was significantly shortened in the multi-injection group $(P<$ 0.05).

Conclusion: By injecting ICG into multiple sites of the affected limbs, we could detect more functional lymphatic vessels during LVA, which was helpful for achieving a successful surgical result.

Keywords: Lymphedema, indocyanine green, indocyanine green lymphography, multipoint injection, lymphaticovenular anastomosis

\section{INTRODUCTION}

Lymphedema is a disease in which lymphatic fluid accumulates in the skin or subcutaneous tissue ${ }^{[1]}$. It usually occurs during tumor surgery, lymph node dissection, or postoperative radiotherapy. The accumulation of protein-rich lymphatic fluid in the skin leads to the infiltration of immune cells and inflammatory response, resulting in chronic inflammation of the subcutaneous tissue. The end results of chronic inflammation are fatty hypertrophy and fibrosis of lymphatic vessels and connective tissue ${ }^{[2]}$.

Lymphaticovenular anastomosis (LVA) is a surgical procedure for the treatment of lymphedema. Because LVA is a minimally invasive procedure, we use LVA as the first choice of surgery. It can effectively reduce the incidence of limb mass, cellulitis, and other complications associated with lymphedema ${ }^{[3-5]}$. However, the standard for LVA has not been established regarding the location, number, and pattern of anastomoses ${ }^{[6]}$. To date, there have been no reports of worsening conditions caused by LVA surgery, even when the procedure was unsuccessful.

Indocyanine green (ICG) is a fluorescent anthocyanin dye that remains in the blood and lymphatic vessels due to its binding to plasma proteins. Now, ICG lymphography is a common method of LVA examination, which is suitable for preoperative, intraoperative, and postoperative evaluation of lymphatic drainage ${ }^{[7-9]}$. It has the unique ability to reveal lymphoid structural disorders and is highly accurate in the assessment of lymphoedema and lymphoid drainage disorders ${ }^{[10]}$. However, when there is no adequate lymphatic function in the distal limb, information about the location of the lymphatic vessels is unobtainable, even if lymphatic vessel function is adequate in the proximal limb. To overcome this limitation, we propose multipoint injection ICG lymphography.

We studied LVA using multipoint injections of ICG. The multipoint injection method could find more functional lymphatic vessels preoperatively, thus improving the successful patency rate of LVA surgery and shortening the operative time. The purpose of this study was to validate the efficacy of LVA combined with multipoint ICG lymphography in the treatment of lymphedema.

\section{METHODS}

\section{Patients}

This study was a prospective study approved by the ethical committee of Xi'an Central Hospital. In total, 42 patients were treated between June 2018 and July 2020, of whom 17 patients received single-point injection of ICG ( 6 cases of upper limbs and 11 cases of lower limbs) and 25 patients received multipoint injection of ICG ( 9 cases of upper limbs and 16 cases of lower limbs). The severity of lymphedema was diagnosed by the 
classification of the International Society of Lymphology (ISL) ${ }^{[11]}$.

\section{ICG lymphography}

We performed ICG lymphography preoperatively for all patients. In the single-point injection group (control group), 0.1 mL ICG was injected subcutaneously into the first web space of each foot or the second web space of the hand. In the multipoint injection group, if we could not observe a linear pattern, four injection points were added in the lower limbs, including the medial and malleolus and the medial and lateral knees, respectively, and two injection points in the lateral wrist and the medial elbow were added in the upper limbs. On the contrary, we injected ICG at only one site if we could find a good linear pattern in both the lower leg and the thigh or both the forearm and the upper arm with the first injection. Therefore, some of the patients in this group had only one injection. The number of injection sites was increased to ensure that a sufficient number of linear lymphatic drainage images could be found. After injection, images of the lymphatic drainage were observed using an infrared camera system and marked on the patient's skin [Figure 1]. The selection of the incision was based on the linear pattern of ICG lymphography. In the control group, if no linear pattern of ICG was observed, the location of the incision was determined based on the anatomical location of the lymphatic vessels, usually along the great saphenous vein ${ }^{[12]}$. In the multiinjection group, a linear pattern of ICG lymphography was usually observed.

\section{The LVA procedure}

All patients underwent LVA surgery under general anesthesia. After administering the anesthetic, the skin was cut with a scalpel in the designed incision, looking for venules in the subcutaneous fat layer and lymphatic vessels beneath the superficial fascia. Matching lymphatic vessels and blood vessels were found and anastomosed end-to-end using 12-0 nylon thread. If matching lymphatic and blood vessels were not found, or if the search took more than $40 \mathrm{~min}$, the incision was abandoned and a new incision was performed and the process started again. We anastomosed the upper limb in three places and the lower limb in four places.

\section{Evaluations and statistical analysis}

The time of each operation and the number of incisions were recorded. Preoperative and postoperative evaluation included the change in circumference of the affected $\operatorname{limb}^{[1]}$. We collected the sum of the circumference of six points of lower limbs (dorsal of foot, ankle, lower edge of patella $10 \mathrm{~cm}$, upper edge of patella $10 \mathrm{~cm}$, upper edge of patella $10 \mathrm{~cm}$, and upper edge of patella $20 \mathrm{~cm}$ ) and the sum of the circumference of the four points of the upper extremity (dorsal hand, wrist, elbow, and $10 \mathrm{~cm}$ above elbow) before and six months after surgery. Each item related to the surgical results and the patients was compared between the control group and the multi-injection group using the Student's $t$-test or the $\chi^{2}$ test. The level of statistical significance was set at $P<0.05$.

\section{RESULTS}

In total, 42 patients were treated between June 2018 and July 2020, of whom 17 patients received singlepoint injection of ICG ( 6 cases of upper limbs and 11 cases of lower limbs) and 25 patients received multipoint injection of ICG (9 cases of upper limbs and 16 cases of lower limbs). The patients' characteristics are shown in Table 1. The average age was 56.3 years old (range: 45-67 years old). All patients were female. There were 15 cases of upper extremity lymphedema and 27 cases of lower extremity lymphedema. The average lymphedema duration was 116.5 months (range: 13-313 months). For both groups, the follow-up was six months. 
Table 1. Characteristics of the patients

\begin{tabular}{|c|c|c|c|c|c|}
\hline & & Total & Multi injection & Control & $\boldsymbol{P}$ \\
\hline Number of patient & & 42 & 25 & 17 & \\
\hline Age (year) & Average (range) & $56.3(45-67)$ & $55.6(45-67)$ & $56.8(47-67)$ & 0.491 \\
\hline \multirow[t]{2}{*}{ Sex } & Female & 42 & 25 & 17 & \\
\hline & Male & - & - & - & \\
\hline \multirow[t]{2}{*}{ The number of lymphedema limbs } & Upper & 15 & 9 & 6 & \\
\hline & Lower & 27 & 16 & 11 & \\
\hline Lymphedema duration (months) & Average (range) & $116.5(13-313)$ & $112.9(13-313)$ & $121.8(21-312)$ & 0.691 \\
\hline \multirow[t]{3}{*}{ ISL classification } & 1 & 12 & 6 & 6 & \\
\hline & 2 & 18 & 11 & 7 & \\
\hline & 3 & 12 & 8 & 4 & \\
\hline
\end{tabular}

ISL: International society of lymphology.

In the multi-injection group, the upper limbs were injected with ICG at an average of 2.2 sites (range: 1-3 sites) and the lower limb at an average of 3.2 sites (range: 1-5 sites). The average number of incisions in the upper limbs of the two groups was four (range: 3-6 in the control group and 3-5 in the multi-injection group) and the lower limb was five in both groups (range: 4-8 in the control group and 4-6 in the multiinjection group). The surgical duration for the upper limb in the control group was $269 \pm 47 \min (P=0.017)$ and in the multi-injection group was $218 \pm 27 \mathrm{~min}$. The surgical time for the lower limb in the control group was $363 \pm 40 \mathrm{~min}$ and in the multi-injection group was $322 \pm 55 \mathrm{~min}(P=0.035)$ [Table 2].

In the control group, the mean preoperative and postoperative circumferences of the upper limbs (sum of four points) were $115.4 \pm 3.2$ and $110.8 \pm 3.3 \mathrm{~cm}(P=0.033)$ and of the lower limbs (sum of six points) were $246.0 \pm 9.8$ and $239.2 \pm 9.9 \mathrm{~cm}$, respectively $(P=0.118)$.In the multi-injection group, the mean preoperative and postoperative circumferences of the upper limbs (sum of four points) were $116.3 \pm 4.8$ and $108.2 \pm 4.0$ $\mathrm{cm}(P=0.001)$ and of the lower limbs (sum of six points) were $248.7 \pm 7.9$ and $236.2 \pm 7.8 \mathrm{~cm}$, respectively ( $P<0.05)$. The circumference change of upper limbs in the control group was $-3.95 \% \pm 1.34 \%$ and in the multi-injection group was $-6.96 \% \pm 0.88 \%(P<0.05)$. The change in circumference of lower limbs was $-5.01 \% \pm 2.2 \%$ in the multi-injection group and $-2.33 \% \pm 1.77 \%$ in the control group $(P=0.003)$ [Figure 2]

\section{DISCUSSION}

In this study, compared with the control group, we could find more effective lymphatic vessels by multiinjection of ICG before LVA. This may significantly improve the results of surgical outcome. According to the results of this study, the indications of LVA may be expanded and more patients may benefit from this low-invasive surgery.

ICG imaging can detect lymph vessel located up to 1-2 $\mathrm{cm}$ below the skin surface. Normal ICG lymphography shows superficial lymph vessels as white lines on a screen dynamically. ICG lymphography can show lymph vessels immediately after injection of ICG. When images of lymph vessels are unclear, the ICG injection site should be massaged manually ${ }^{[13]}$.

Sometimes, the injury of a lymphatic vessel starts at the distal side of the limb and the contrast agents cannot enter the lymphatic vessels, so the lymphatics cannot be seen when these substances are injected into the dorsal side of the foot or hand ${ }^{[14]}$. ICG lymphography findings are largely classifiable into two patterns: linear pattern and dermal backflow pattern ${ }^{[15]}$. Unfortunately, many patients present with dermal backflow pattern at their initial visit. In clinical work, we inject additional ICG into the medial and lateral side of the 
Table 2. Surgical results

\begin{tabular}{|c|c|c|c|c|c|}
\hline & & Total & Multi injection & Control & $\mathbf{P}$ \\
\hline \multirow{2}{*}{$\begin{array}{l}\text { Number of injection per limb } \\
\text { Average (range) }\end{array}$} & Upper & & $2.2(1-3)$ & 1 & \\
\hline & Lower & & $3.2(1-5)$ & 1 & \\
\hline \multirow{2}{*}{$\begin{array}{l}\text { Number of incision per limb } \\
\text { Average (range) }\end{array}$} & Upper & 4 & $3.7(3-5)$ & $4.5(3-6)$ & 0.117 \\
\hline & Lower & 5 & $4.6(4-6)$ & $5.5(4-8)$ & 0.058 \\
\hline \multirow[t]{2}{*}{ Number of LVA per limb } & Upper & 3 & 3 & 3 & \\
\hline & Lower & 4 & 4 & 4 & \\
\hline \multirow[t]{2}{*}{ Operation time per patient ( $\mathrm{min}$ ) } & Upper & $238(180-324)$ & $218(180-260)$ & $269(196-324)$ & 0.017 \\
\hline & Lower & $339(265-450)$ & $322(265-405)$ & $363(286-450)$ & 0.035 \\
\hline
\end{tabular}

LVA: Lymphaticovenular anastomosis.

ankle and the knee, when we cannot find a linear pattern after injecting ICG into the dorsum of the foot. In this study, we could find more lymphatic vessels and the surgical results were better than the results in the control group. Preoperative detection of functional lymphatics is an important aspect of a successful LVA. For this purpose, we applied multi-injection ICG to detect the lymphatic vessels.

Mihara et al. ${ }^{[16]}$ reported that lymphatic vessels of the ectasis type was most effective for LVA. In this study, the proportion of the ectasis type lymphatic vessels in the multi-injection group was significantly higher than that in the control group, indicating that more ectasis type lymphatic vessels could be detected by multi-injection ICG. Although the number of anastomoses was similar between the two groups, more Ectasis type lymphatic vessels were found in the multi-injection group, which could explain the improved surgical outcomes. Therefore, injecting ICG into the distal part of the extremities as a preoperative examination for LVA was insufficient.

In this study, the surgical duration of the limb was significantly shortened in the multi-injection group. Based on the linear pattern of the ICG lymphogram, we determined the surgical incision. When we could not observe a linear pattern, we made an incision along the anatomical location of the lymphatic vessels, which was usually along the pattern of the great saphenous vein. However, it was not always possible to accurately detect the patient's lymphatic vessels, and thus more time was sometimes required to find the lymphatic vessels during the surgery. By the multi-injection method, we were able to locate more linear patterns than in the control group. This technique could offer significant help to the surgeon in rapidly locating the lymphatic vessels during surgery.

Multiple injections of ICG lymphography could help us find more functional lymphatics than when using conventional ICG lymphography. We were sometimes able to observe linear patterns in the limb when the ICG was injected into more sites, even if we could not see the spread of the ICG injected into the dorsum of the hand or foot.

To ensure the same observation time between the two groups, the limitation of this study was that the follow-up time was not long enough. Based on our experience, lymphedema usually continues to improve in patients who improve after six months. Prolonged postoperative follow-up is necessary to assess the longterm effects of LVA.

In conclusion, multipoint injection ICG in lymphography could effectively improve the efficacy of LVA. It could help us to detect more functional lymphatic vessels suitable for anastomosis. The proportion of ectasis type lymphatic vessels used for anastomosis in the multi-injection group was significantly higher, which 

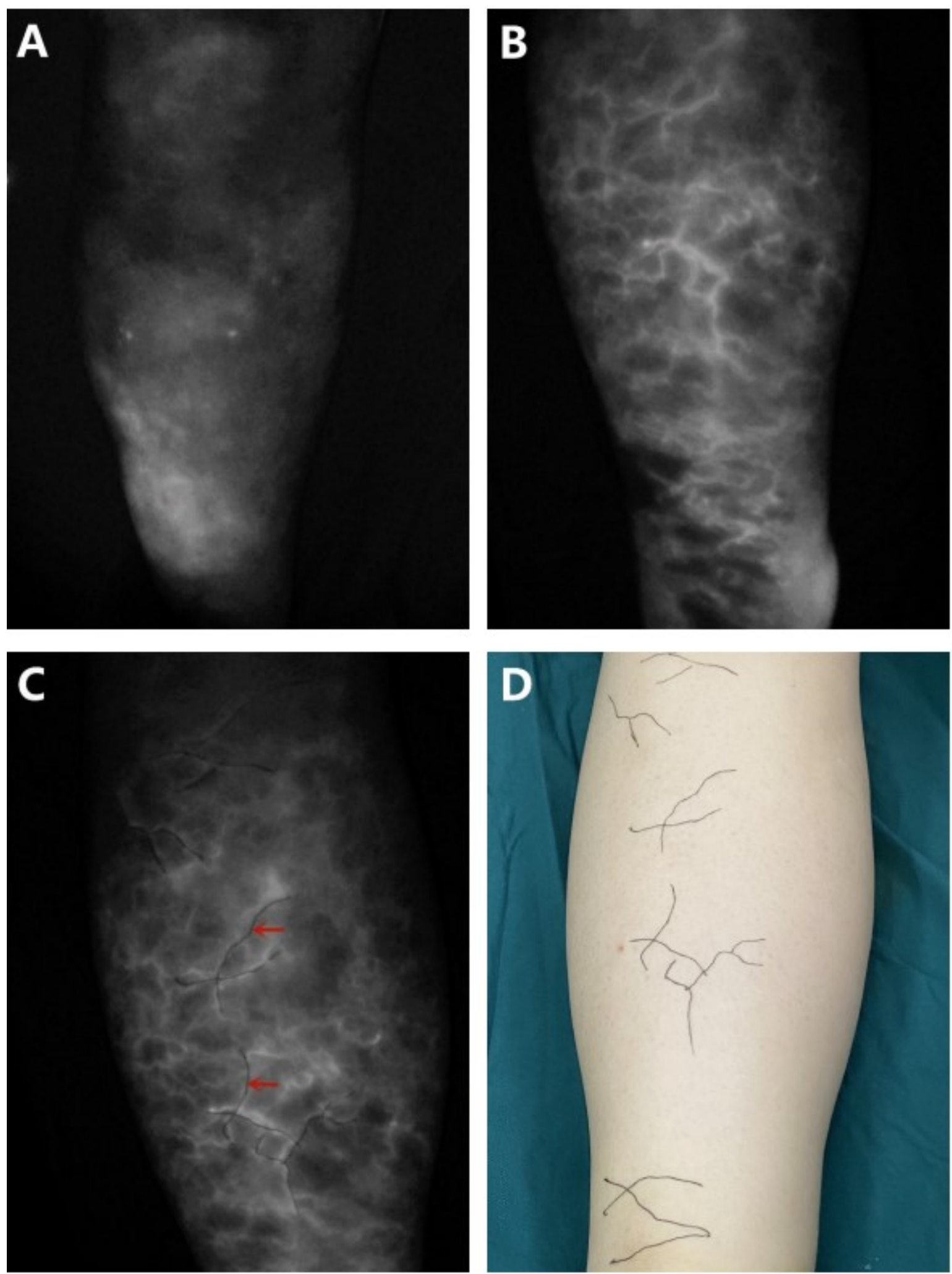

Figure 1. Images of the lymphatic drainage were observed using an infrared camera system and marked on the patient's skin: (A) control group, indocyanine green-lymphangiography was performed; (B) multi-injection group, more linear patterns of ICG lymphography were found; (C) multi-injection group, the indocyanine green lymphangiography findings were marked on the skin (red arrow); and (D) the patient had a linear pattern on the lower limb. ICG: Indocyanine green. 


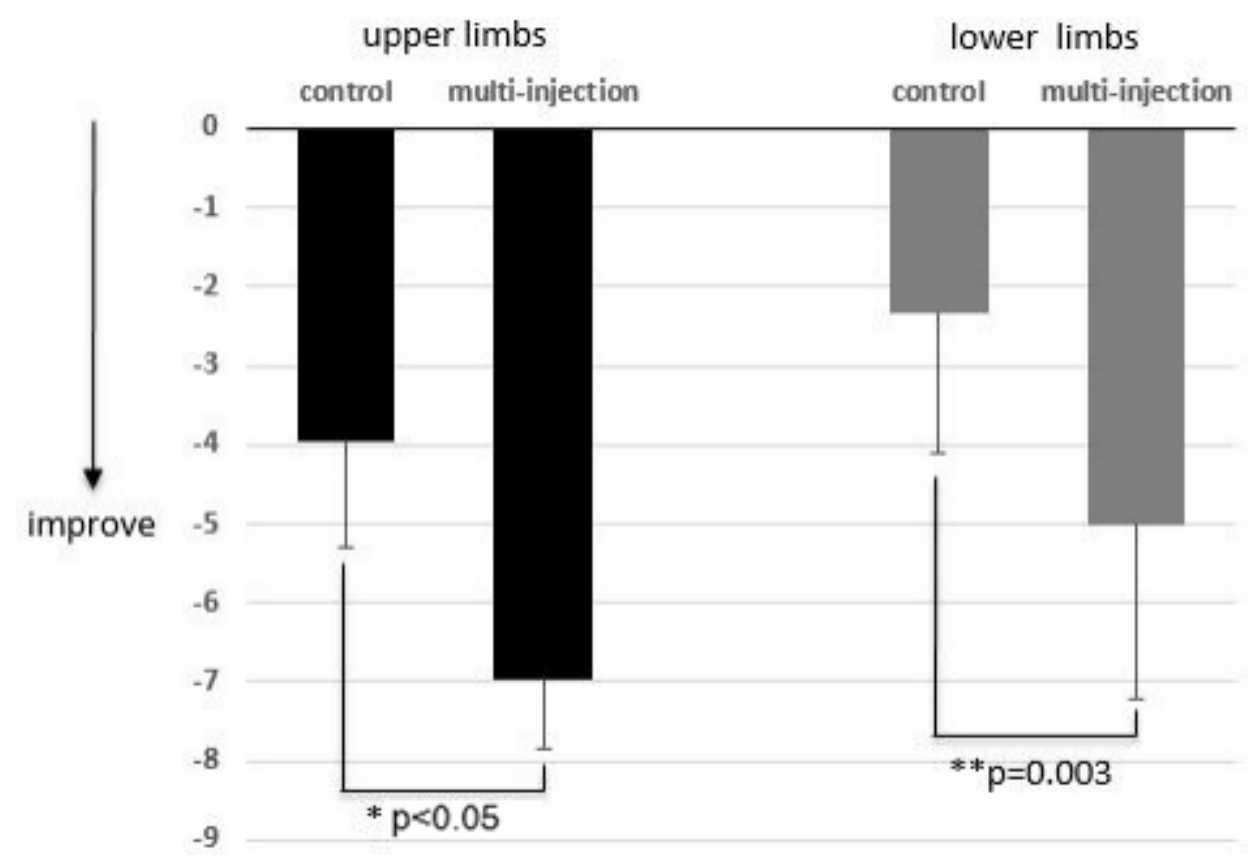

Figure 2. Comparison of the circumference change between the control group and the multi-injection group. Negative numbers indicate improvement. The circumference change of the upper limbs in the control group was $-3.95 \% \pm 1.34 \%$ and in the multi-injection group was $-6.96 \% \pm 0.88 \%\left({ }^{\star} P<0.05\right)$. The change in circumference of the lower limbs was $-5.01 \% \pm 2.2 \%$ in the multi-injection group and $-2.33 \% \pm 1.77 \%$ in the control group $\left({ }^{\star \star} P=0.003\right)$.

may have contributed to shortening the surgical duration and the circumference reduction. Multipoint injection ICG in lymphography is helpful for preoperative assessment of lymphatic function and determination of lymphatic function in the whole limb.

\section{DECLARATIONS}

Authors' contributions

Made substantial contributions to conception and design of the study and performed operation: Liu B, Ma G

Provided technical, and material support: Han Y, Liu B

Performed operation and data acquisition: Ma G, Cui X, Wang W

\section{Availability of data and materials}

Not applicable.

\section{Financial support and sponsorship}

None.

\section{Conflicts of interest}

All authors declared that there are no conflicts of interest.

\section{Ethical approval and consent to participate}

The ethical committee of Xi'an Central Hospital, Medical School of Xi'an Jiaotong University approved this study (LW-2021-011). 


\section{Consent for publication}

A written informed consent was obtained from all patients.

\section{Copyright}

(c) The Author(s) 2021.

\section{REFERENCES}

1. Hara H, Mihara M. Multi-area lymphaticovenous anastomosis with multi-lymphosome injection in indocyanine green lymphography: a prospective study. Microsurgery 2019;39:167-73. DOI PubMed

2. Liu HL, Wong MMK, Chung JHP. Indocyanine green lymphangiography-guided liposuction in breast cancer-related lymphedema treatment - patient selection and technique. Plast Aesthet Res 2020;7:6. DOI

3. Hara H, Mihara M. Multilymphosome injection indocyanine green lymphography can detect more lymphatic vessels than lymphoscintigraphy in lymphedematous limbs. J Plast Reconstr Aesthet Surg 2020;73:1025-30. DOI PubMed

4. Mihara M, Hara H, Zhou HP, Tange S, Kikuchi K. Lymphaticovenous anastomosis releases the lower extremity lymphedemaassociated pain. Plast Reconstr Surg Glob Open 2017;5:e1205. DOI PubMed PMC

5. Mihara M, Hara H, Furniss D, et al. Lymphaticovenular anastomosis to prevent cellulitis associated with lymphoedema. Br J Surg 2014;101:1391-6. DOI PubMed

6. Campisi CC, Ryan M, Boccardo F, Campisi C. A single-site technique of multiple lymphatic-venous anastomoses for the treatment of peripheral lymphedema: long-term clinical outcome. J Reconstr Microsurg 2016;32:42-9. DOI PubMed

7. Yamamoto T, Narushima M, Doi K, et al. Characteristic indocyanine green lymphography findings in lower extremity lymphedema: the generation of a novel lymphedema severity staging system using dermal backflow patterns. Plast Reconstr Surg 2011;127:1979-86. DOI PubMed

8. Hara H, Mihara M. Blocking of the lymphatic vessel in lymphedema. Eplasty 2017;17:e11. PubMed PMC

9. Mihara M, Hara H, Shibasaki J, et al. Indocyanine green lymphography and lymphaticovenous anastomosis for generalized lymphatic dysplasia with pleural effusion and ascites in neonates. Ann Vasc Surg 2015;29:1111-22. DOI PubMed

10. Guermazi A, Brice P, Hennequin C, Sarfati E. Lymphography: an old technique retains its usefulness. Radiographics 2003;23:154158; discussion 1559. DOI PubMed

11. Society of Lymphology. The diagnosis and treatment of peripheral lymphedema: 2013 consensus document of the international society of lymphology. Lymphology 2013;46:1-11. PubMed

12. Pan WR. Atlas of lymphatic anatomy in the head, neck, chest and limbs. Singapore: Springer; 2017. DOI

13. Narushima M, Yamamoto T, Ogata F, Yoshimatsu H, Mihara M, Koshima I. Indocyanine green lymphography findings in limb lymphedema. J Reconstr Microsurg 2016;32:72-9. DOI PubMed

14. Hara H, Mihara M. Lymphaticovenous anastomosis for advanced-stage lower limb lymphedema. Microsurgery 2021;41:140-5. DOI PubMed

15. Sty JR, Boedecker RA, Scanlon GT, Babbitt DP. Radionuclide “dermal backflow” in lymphatic obstruction. J Nucl Med 1979;20:9056. PubMed

16. Mihara M, Hara H, Tange S, et al. Multisite lymphaticovenular bypass using supermicrosurgery technique for lymphedema management in lower lymphedema cases. Plast Reconstr Surg 2016;138:262-72. DOI PubMed 